\title{
Linear Algebra Representation of Necker Cubes I I: The Routley Functor and Necker Chains
}

\author{
Chris Mortensen \\ Dept of Philosophy \\ The University of Adelaide \\ North Terrace, SA 5005 \\ Australia
}

Chris.Mortensen@adelaide.edu.au

Received by Greg Restall

Published March 27, 2009

http://www.philosophy.unimelb.edu.au/ajl/2009

(C) 2009 Chris Mortensen

\begin{abstract}
In this sequel, linear algebra methods are used to study the Routley Functor, both in single Neckers and in Necker chains. The latter display a certain irreducible higher-order inconsistency. A definition of degree of inconsistency is given, which classifies such inconsistency correctly with other examples of local and global inconsistency.
\end{abstract}

\section{INTRODUCTION}

This paper is a sequel to Mortensen and Leishman [2009]. In the first section, I proceed to an analysis of the role of the Routley Functor on inconsistent and incomplete theories generated by the simplest case of a single Necker cube. It is shown here that the Routley Functor is definable in terms of linear-algebraic operations on matrices, and conversely, and that the Routley functor commutes with various combinations of these operations. These results constitute a further application of the Routley Functor outside its usual home, and contribute further to its explanation and justification. In the second section, I show how to extend the application of linear algebra to chains of Neckers displaying a certain higher-order inconsistency. This enables a definition of degree of inconsistency which is a natural generalisation of the discussion in [2009].

In the previous paper, it is shown how to represent the various consistency and completeness properties of Necker cubes by associating each Necker with a matrix, called the primary matrix $M_{p}$, over the field $Z_{2}$. Locally inconsistent Neckers were those with at least one row of $M_{p}$ being a pair of ones $[1,1]$, incomplete Neckers were those with at least one row being a pair of zeros $[0,0]$, and the two globally inconsistent Neckers (the Crazy Crates, also called 
the Escher cubes) were represented either the diagonal matrix $\operatorname{Id}=\left[\begin{array}{ll}1 & 0 \\ 0 & 1\end{array}\right]$ or by the anti-diagonal matrix Anti-Id $=\left[\begin{array}{ll}0 & 1 \\ 1 & 0\end{array}\right]$.

The former is standardly called Id in matrix algebra because it is the multiplicative identity, that is both left multiplication and right multiplication by Id leaves a matrix unchanged. We see the properties of Anti-Id as we go along.

\section{THE ROUTLEY FUNCTOR}

\section{I ELEMENTARY OPERATIONS ON MATRICES}

It will be recalled from linear algebra that on any matrix there are three elementary row operations, these being:

(I) RowSwap (swap a pair of rows)

(2) RowAdD (add one row to another)

(3) ScalarMultiply (multiply a row by a non-zero scalar)

It is noted that in $Z_{2}$, (3) is redundant, since the only non-zero scalar is 1 . Furthermore, for $2 \times 2$ matrices, Row Swap and RowADD take single matrices as arguments since there is only one pair of rows to be operated on. But RowADD comes in two variants:

(4) RowAdD1 (add one row to another and put the result in row 1)

(5) RowAdD2 (ditto but put the result in row 2)

Now we can see:

THEOREM I Let $\mathrm{M}$ be any $2 \times 2$ matrix over $\mathrm{Z}_{2}$. Then:

(a) RowSwap $(M)=$ Anti-Id $\times M$

(b) $\operatorname{RowAdd} 1(M)=\left[\begin{array}{ll}1 & 1 \\ 0 & 1\end{array}\right] \times M$

(c) $\operatorname{RowAdD} 2(M)=\left[\begin{array}{ll}1 & 0 \\ 1 & 1\end{array}\right] \times M$

Proof: These can be verified by straightforward matrix multiplication.

Now we can identify further operations, particularly the column duals ColSwap (swap the columns), ColAdd1 (add the columns and put the result in column 1) and ColADD2 (add the columns and put the result in column 2). It can now be proved that: 
THEOREM 2 (a) $\operatorname{CoLSwap}(M)=M \times A n t i-I d$

(b) $\operatorname{ColAdd} 1(M)=M \times\left[\begin{array}{ll}1 & 0 \\ 1 & 1\end{array}\right]$

(c) $\operatorname{ColAdd} 2(M)=M \times\left[\begin{array}{ll}1 & 1 \\ 0 & 1\end{array}\right]$

Proof: These may likewise be verified by straightforward matrix multiplication.

One more matrix operation (not elementary) is Swiтch. This is the result of reversing all $1 \mathrm{~s}$ and $0 \mathrm{~s}$ in the matrix $M$. Think of Sw ITCH as the maximal switching act on an $n \times n$ array of switches.

THEOREM $3 \mathrm{SWITCH}(M)=M+\mathrm{I}$, where $\mathrm{I}$ is the $2 \times 2$ matrix all of whose entries are $1 s$.

Proof: Follows from the fact that in $Z_{2}, 0+1=1$ and $1+1=0$.

\subsection{THE ROUTLEY STAR ON MATRICES}

We return to the characterisation in Section 2 of [2009] of a locally inconsistent Necker as having a primary matrix with at least one row being $[1,1]$. This arises because we identify matrices as being locally inconsistent if they have both colours $R, B$ on at least one crossing, say $C_{1}$. As noted in 2006 , this is not inconsistent by itself, but it is inconsistent if one has as part of one's theory the axiom of local consistencyLoCon: $(\mathrm{C})(\mathrm{R} @ \mathrm{C} \rightarrow \neg \mathrm{B} @ \mathrm{C})$, along with its equivalent: $(C)$ (B@C $\rightarrow \neg R @ C)$. This means that the property of local consistency at the crossing $C_{i}$ can be described in terms of the equations: $a \cdot R+b \cdot \neg R=C_{i}$. Then we can form what can be called the inconsistency equation for a single Necker:

$$
M_{p} \times\left[\begin{array}{c}
R \\
\neg R
\end{array}\right]=\left[\begin{array}{l}
C_{1} \\
C_{2}
\end{array}\right]
$$

where $M_{p}$ is its primary matrix. Clearly this is the primary equation with $\neg R$ substituted for $B$, but it has the merit of displaying explicitly the local inconsistency when Axiom LoCon is added.

If $a=b=1$ we have the formal local inconsistency of having both $R$ and $\neg R$ at $C_{i}$. This representation justifies in a more direct way the claim in [2009]: that local inconsistency in the principal matrix $M_{p}$ and its associated Necker, comes to having a $[1,1]$ row. Correspondingly, a row $[0,0]$ indicates that the theory lacks both R@C and $\neg \mathrm{R} @ C$ for the corresponding crossing C. That is, if $a=b=0$, we have the formal incompleteness of the theory. This also justifies in a more direct way the claim in [2009], that incompleteness in the primary matrix and its associated Necker comes to having a $[0,0]$ row. 
We recall that, if $S$ is any set of sentences, then $S^{*}$ is $\operatorname{defined}$ as $\{A: \neg A \notin S\}$. Now the star of an inconsistent theory is an incomplete theory, and vice versa. Reflected in the primary matrix, this motivates the definition:

Definition 4 The Routley Functor * operating on matrices $M$, is the operation that switches all $[0,0]$ rows to $[1,1]$ rows, and vice versa, and leaves untouched all other rows.

Now we have:

Theorem $5^{*}=$ Switch.ColSwap $=$ ColSwap.Switch.

Proof: Consider the first equation. There are 4 cases, being four possible rows. Consider a row $[0,1]$. ColSwap produces $[1,0]$ and then Switch produces $[0,1]$, which is unchanged. The row $[1,0]$ is similar. Applied to the row $[1,1]$ ColSwap gives $[1,1]$ which when Switched gives $[0,0]$. Finally, ColSwap $[0,0]=[1,1]$ which when Switched gives $[0,0]$. Hence ${ }^{*}=$ Sw itch.ColSwap. The second equation is a similar argument.

That is, Switch commutes with ColSwap, and their product is the Routley Functor, which is thus definable in those terms. Also:

TheOREm $6 *(\mathrm{M})=(\mathrm{M}+\mathrm{I}) \times$ Anti-Id $=(\mathrm{M} \times$ Anti-Id $)+\mathrm{I}$

Proof: From Theorem 3 , adding I has the effect of Sw ITCH; the theorem then follows by applying Theorem 2

Theorem 7 (a) Switch $={ }^{*}$.ColSwap $=$ ColSwap. ${ }^{*}$

(b) ColSwap $={ }^{*} . S$ w ITCH $=$ Sw itch. ${ }^{*}$

Proof: Part (a): It suffices that the equation holds for each row. So begin with any row of the form $[1,0]$. ColSwap produces $[0,1]$ which ${ }^{*}$ leaves unchanged. The case $[0,1]$ is similar. ColSwap $[1,1]=[1,1]$ then applying * gives $[0,0]$. The case $[0.0]$ is similar. The proof of Part (b) is similar.

Thus, from Theorems 5 and 7 , the operators *, ColSwap and Switch form a pairwise commuting triplet such that each is the product of the other two. It is obvious that all operators are involutions. Hence we have that Id $=$ Switch $^{2}={ }^{* 2}=$ ColSwap $^{2}=$ Switch. ${ }^{*}$.ColSwap etc. The three operators thus enjoy a close relationship, which further vindicates the Routley * as a natural operation on these matrices. In the next section, it will be seen that this relationship is continued when moving to larger figures and matrices. DEFinition 8 Anti-* $^{*}$ is the operation that does to columns what Routley * does to rows, that is Anti-* switches $[0,0]$ columns to $[1,1]$ columns and vice versa. 
Theorem 9 (a) $A n t i{ }^{*}=$ Switch.RowSwap $=$ RowSwap.Sw ITCH

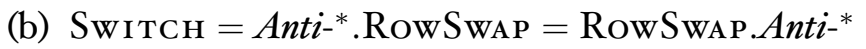

(c) RowSwap $=A n t i-{ }^{*} \cdot \operatorname{Switch}=\operatorname{Switch} \cdot A n t i-*$

(d) $I d=\mathrm{SwITCH}^{2}=A n t i-{ }^{2}=\operatorname{Row} \mathrm{SwaP}^{2}=\mathrm{SwITCH} \cdot$ Anti- $^{*}$.RowSwaP etc.

Proof: These can all be verified by matrix calculation, or by symmetry from the previous theorem. For example, part (a): It sufficies to prove the equation for each column. So begin with any column of the form of the column vector $\operatorname{col}[1,1]$. Applying RowSwap gives col $[1,1]$ and then applying Sw Itch gives $\operatorname{col}[0,0]$ which is Anti- $(\operatorname{col}[1,1])$. The argument for $\operatorname{col}[0,0]$ is similar. Next take col[1,0]. Applying RowSwap gives col $[0,1]$ then applying $S$ witc $H$ gives $\operatorname{col}[1,0]$ which we began with. But this is the same as Anti-*, which leaves such rows unchanged. The argument for $\operatorname{col}[0,1]$ is similar.

This means that the Routley Functor has a natural dual in the dual space, which is definable in terms of SwIтcH and the elementary row operation RowSwap . Again we see an example of three operations closely related. The previous results can now be combined, in virtue of the fact that SwITCH is common to both spaces, to give results like:

TheOrem io (a) ${ }^{*}$.ColSwap $=A n t i-{ }^{*}$.RowSwap

(b) ${ }^{*}=\mathrm{SwITCH} .{ }^{*} . \mathrm{SwitCH}$

\subsection{TRANSPOSES}

We recall the well-known matrix operator Transpose, that is $\mathrm{T}$, defined in [2009] as the result of swapping rows for columns across the main diagonal, ie. element $a_{i j}$ becomes element $a_{j i}$. Transpose is clearly an involution, that is $M^{\mathrm{TT}}=M$. We can also define Anti-Transpose, shortened to Anti-T, as the operation which swaps rows and columns across the anti-diagonal. Now we have:

THEOREM II (a) RowSWAP.T = T.COLSwap, and similarly with ANTI-T for $\mathrm{T}$

(b) RowSwap $=$ T.ColSwap.T, and similarly for ANTI-T

(c) $\mathrm{T}=$ RowSwap.T.ColSwap, and similarly for ANTI-T

(d) $A n t i{ }^{*} \cdot \mathrm{T}=\mathrm{T}{ }^{*}$

(e) $A n t i{ }^{*}={ }^{*}{ }^{*} \cdot \mathrm{T}$

(f) Switch.T = T.Switch 
(g) Anti-Id $=\operatorname{Switch}(I d)=\operatorname{RowSWap}(I d)=\operatorname{ColSwap}(I d)$.

(h) $A n t i-I d^{2}=I d$

Proof: For (a), we have: From $\left[\begin{array}{ll}a & b \\ c & d\end{array}\right]$ first applying CoLSwap gives $\left[\begin{array}{ll}b & a \\ d & c\end{array}\right]$ then applying $T$ gives $\left[\begin{array}{ll}b & d \\ a & c\end{array}\right]$ whereas first applying $T$ gives $\left[\begin{array}{ll}a & c \\ b & d\end{array}\right]$ then applying RowSwap gives the same $\left[\begin{array}{ll}b & d \\ a & c\end{array}\right]$.

Part (b) follows by applying $T$ on the right to both sides of (a). Part (c) follows by applying CoLSwap on the right to both sides of (a). For Part (d), it is clear that first swapping $[1,1]$ rows for $[0,0]$ rows then transposing, is the same as first transposing, then swapping $[1,1]$ columns for $[0,0]$ columns. Part (e) follows from (d) by application of $\mathrm{T}$ on the right. Parts (f) and (g) are obvious. Part (h) may be verified by matrix multiplication.

COMment Note, in contrast with $(\mathrm{d})$, that in general $\mathrm{T} .{ }^{*} \neq{ }^{*}$. $\mathrm{T}$, that is $\mathrm{T}$ and Star do not commute; this can be seen by applying these succssively to $\left[\begin{array}{ll}1 & 1 \\ 0 & 0\end{array}\right]$.

\subsection{ORDER, COVARIANCE AND CONTRAVARIANCE}

There is a natural order on matrices:

DEFINITION I2 For matrices $M, N$ we can define $M \leqslant N$ to mean that each element of $M$ is $\leqslant$ the corresponding element of $N$, and $M<N$ to mean $M \leqslant N$ and $M \neq N$. An operation is said to be Covariant if it is orderpreserving, and CONTRAVARIANT if it is order-reversing.

The question then arises: which of the above operations are covariant and which contravariant?

THEOREM I3 $M<N$ iff Switch $(\mathrm{N})<\operatorname{SwITCH}(M)$ (Switch is contravariant).

Proof: If $M<N$ then $M$ has 0 s in some places where $N$ has $1 \mathrm{~s}$, and they otherwise agree. Hence $\operatorname{Switch}(N)$ has 0 in some places where $\operatorname{Switch}(M)$ has 1 s and they otherwise agree. Hence $\operatorname{Switch}(N)<\operatorname{Switch}(M)$.

THEOREM I4 The following are covariant: RowSwap, ColSwap, T, Anti-T.

Proof: It is evident that these operations preserve the order as they preserve which entry corresponds to which. 
THEOREM I5 The Routley * and Anti-* are contravariant.

Proof: That the Routley * is contravariant follows from Theorem 5 , namely that ${ }^{*}=$ Switch.ColSwap. That is, ColSwap is order-preserving, and then Switch reverses the order. That Anti-* is contravariant is a similar argument. Alternatively, we may invoke Theorem II(e), namely Anti- ${ }^{*}=\mathrm{T}^{*} . \mathrm{T}$, for then $\mathrm{T}$ preserves order, ${ }^{*}$ reverses it, and finally $\mathrm{T}$ again preserves it.

It is an appropriate result that the Routley Functor on matrices is contravariant, since its counterpart on theories is order-reversing under the subset relation $\subset$ as order. But the derivation of this fact from the contravariance of SwITCH together with the covariance of other matrix operations is novel, as is the dual result for Anti-* ${ }^{*}$ These results demonstrate the applicability and richness of the Routley Functor interacting with linear algebra, which adds to the motivation that we have seen for it up to now.

In the next section, these methods are extended to the representation of arbitrary collections of Neckers, and the special case of several Neckers chained together. It is seen that some modification is necessary, but also that some of the representations of the Routley Functor remain invariant.

\section{NECKER CHAINS}

I begin with an obvious point, that the most general case of $n$ Neckers, coloured differently and distributed randomly across a page, does not have much interest beyond what has already been shown: they are simply described by $n$ matrices, each $2 \times 2$. These could be arranged artificially into a single $2 n \times 2 n$ matrix over $Z_{2}$, but its $2 \times 2$ cells would have no interaction with one another. The possible diagrams that can be drawn with $n$ Neckers are too diverse to get much theoretical purchase on, beyond the case of the single Necker.

However, there is a more interesting special case which permits an additional higher order inconsistency. This higher order inconsistency is itself a generalisation of the global inconsistency of the previous section, in that both arise from incompatibility between structures that are consistent in themselves. The special case we study is the case where $n$ Neckers of similar colours are chained together in a row. We can call this an N-CHAIN, and each Necker from which it is constructed is called a CELL. It should be stressed that the colouring of the Neckers is not essential. As was seen in 2006, it serves only as a convenient vehicle for disambiguation of the individual Neckers, that is the case $n=1$, by keeping track of faces, which can in any case be achieved by the device of breaking the occluded edge as in knot theory. Such higher order inconsistency is manifested in the case $n=2$, as below, where each individual Necker cell is entirely consistent, only the relation between the two is impossible. We use this $n=2$ case as an illustration of the results that follow. 


\section{I CHAINED NECKERS}

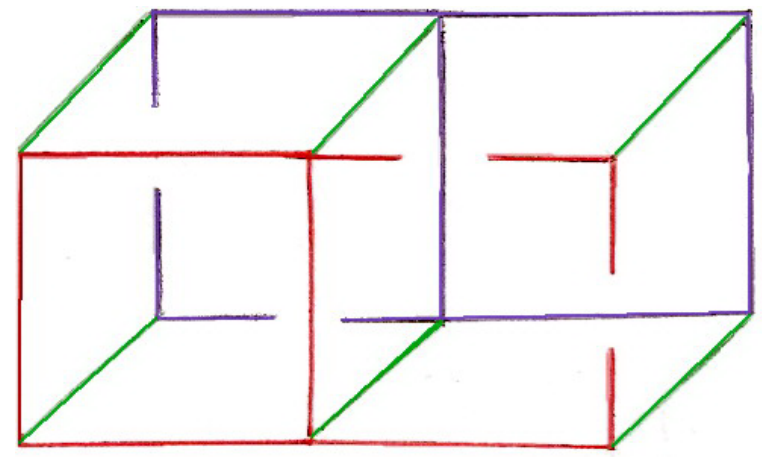

Figure I: Higher Order Inconsistency

Any 2-chain of Neckers, for example the above figure, has four crossings. As in earlier papers, each crossing can have both colours (local inconsistency), neither (local incompleteness), or one (local consistency and completeness). It is clear that the whole pattern of crossings for any 2-chain can be described in the following matrix equation:

$$
\left[\begin{array}{ll}
a & b \\
c & d \\
e & f \\
g & h
\end{array}\right] \cdot\left[\begin{array}{l}
R \\
B
\end{array}\right]=\left[\begin{array}{l}
C_{1} \\
C_{2} \\
C_{3} \\
C_{4}
\end{array}\right]
$$

The left hand $4 \times 2$ matrix can be called the primary matrix $M_{p}$ of the diagram. It consists of two $2 \times 2$ cells arranged vertically, each being the primary matrix of the corresponding single Necker cell of the diagram. The same information can be displayed in four individual equations:

$$
\begin{aligned}
\mathrm{aR}+\mathrm{bB} & =\mathrm{C}_{1} \\
\mathrm{cR}+\mathrm{dB} & =\mathrm{C}_{2} \\
\mathrm{e} \mathrm{R}+\mathrm{fB} & =\mathrm{C}_{3} \\
\mathrm{gR}+\mathrm{hB} & =\mathrm{C}_{4}
\end{aligned}
$$

All of the coefficients $a \ldots h$ come from $Z_{2}$; this is all that is necessary since each crossing is formed from the contribution of just two colours. It is also noted that for the general case of $n$ Neckers chained horizontally, there are $2 n$ crossings and a primary matrix of dimension $2 n \times 2$.

In moving to the secondary matrix, the appropriate generalisation takes into account the contributions of red and blue crossings. We recall that in the $n=1$ case, the secondary matrix $M_{s}$ was the transpose of the primary matrix, but that is not available here because the primary matrix is not square. However, we can proceed via an intermediate construction which is available. 
So each of the two Neckers, taken separately, makes up a red face RF and a blue face $B F$, so there are 4 faces, RF1 and BF1, and RF2 and BF2. In the $n=1$ case, that is the $2 \times 2$ construction of [2009], these are described by the secondary equation $M p^{T} . C=F$, where $C$ is the $2 \times 1$ column vector of crossings, and $F$ is the $2 \times 1$ column vector of faces. In moving to the case $n=2$ it is trivial that we can stack the two primary matrices one above the other:

$$
\left[\begin{array}{ll}
a & c \\
b & d \\
e & g \\
f & h
\end{array}\right] \cdot\left[\begin{array}{l}
C_{1} \\
C_{2} \\
C_{3} \\
C_{4}
\end{array}\right]=\left[\begin{array}{l}
R F 1 \\
B F 1 \\
R F 2 \\
B F 2
\end{array}\right]
$$

The left hand matrix is evidently:

$$
\left[\begin{array}{l}
M_{p} 1^{T} \\
M_{p} 2^{T}
\end{array}\right]
$$

But now, proceeding in a linear fashion, we can postulate that the large red face, as the composite of the red faces, is given by the sums of their crossings, and hence the sums of their faces: RF $=R F 1+R F 2$. Similarly for the blue face is the sum of individual blue faces: $\mathrm{BF}=\mathrm{BF} 1+\mathrm{BF} 2$. It is clear how to write this in a matrix equation: it is simply:

$$
\left[M_{p} 1^{T}, M_{p} 2^{T}\right] . C=F
$$

where the (left hand) matrix is the secondary matrix $M_{s}$ having dimensions $2 \times 4$, $C$ is the $4 \times 1$ column vector of crossings and $F$ is the $2 \times 1$ column vector of large faces. This is the secondary equation for the chain of Neckers. In the case of $n$ Neckers, under the assumption that faces add linearly, the secondary matrix is $2 \times 2 n, C$ is $2 n \times 1$, and $F$ remains the same.

The results of [2009] point directly to what is important for inconsistency, either local or higher order (including global). It is the solutions of the unit equation, ie. the set of solutions for $C$ in the case where $F$ is the unit vector $\mathbf{I}$ of all ones. In particular, we want to show that a Necker chain is inconsistent iff its unit equation has solutions; and equivalently, a chain is consistent iff its unit equation has no solutions.

To get to this, first we need a sense of which Necker chains are consistent and which are inconsistent. Every consistent Necker chain must have all its component cells consistent, since if any cell is inconsistent then the whole has that as an inconsistent part. (The reverse fails, as we can see from the above Necker chain with 2 cells, which has each of its component cells consistent, they are simply chained together in an inconsistent way, in which the blue face dominates in one case but the red face dominates in the other.) There are two basic types of consistent Necker chains. (I) There are the two basic consistent Neckers, one in which all red faces and edges are unambiguously in front of the blue faces and edges where they occlude, and the other in which it is blue that 
dominates. (2) In addition, there are all those which can be obtained from (I) by the deletion of one or more colours at a crossing. These are incomplete, since they have crossings at which there are no colours; but they remain consistent, since they are deletions from cases which are all ready consistent. See the figure below for two consistent Neckers, one complete, the other incomplete.
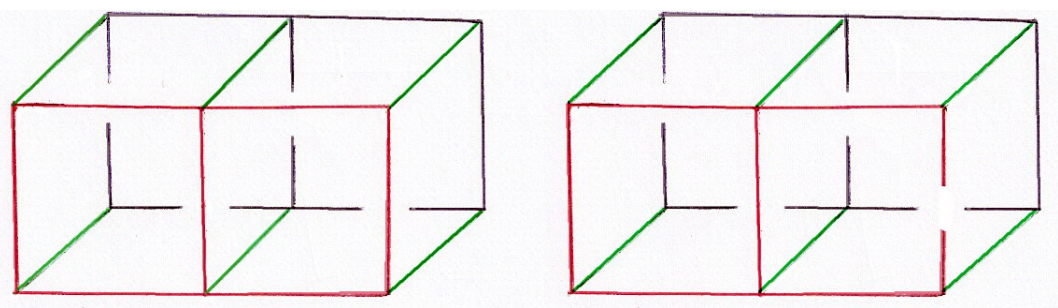

Figure 2: Two Consistent Neckers: One Complete, One Incomplete

All the Necker chains other than types (I) and (2) are inconsistent, and they come in two further forms (3) Red and blue edges both appear at the same crossing. These are inconsistent in virtue of having an inconsistent part, namely that individual crossing. Alternatively, (4) Red and blue never appear at the same crossing, but red is unambiguously in front at one crossing, and blue is unambiguously in front at another crossing. This is inconsistent because no large face can be put together which has the same colour unambiguously in front along the whole chain. The figure we began with is an example. It is clear that these four possibilities are exclusive and exhaustive.

Now we need a lemma

LEMMA 16 The unit equation has no solutions iff one or both of the rores of $\mathrm{M}_{\mathrm{S}}$ is all zeros.

Proof: $\mathrm{R}$ to $\mathrm{L}$ : if one of the rows of $\mathrm{M}_{\mathrm{s}}$ is all zeros, there is no way to assign numbers to the column vector $C$ of crossings to produce a 1 in that row of $F$. Hence the secondary equation has no solutions. L to R: Conversely, suppose that neither row is all zeros. So each row has at least one 1 in it. To find a solution to the secondary equation, (a) find the first column from the left that is both $1 \mathrm{~s}$, and put a 1 in that row of $C$, then complete $C$ with zeros. It is clear that this $C$ corresponds to the pair of equations $0+0+\ldots+1.1+0+\ldots+0=1$ for the first row, and identically for the second row, which is a solution. Otherwise (b), find the first 1 in the first row, say column $i$, then the first 1 in the second row, which must be in a different column $j$ from that in the first row. Put $1 \mathrm{~s}$ in each of the corresponding rows $i, j$ of $C$, and complete $C$ with zeros. This corresponds to the pair of equations $0+0+\ldots+1.1$ (ith place) $+0 \ldots+0=1$, and $0+0+\ldots+1.1(j$ th place $)+0 \ldots+0=1$. Again this is a solution. 
Now we have the main theorem:

THEOREM I 7 A chain of Neckers is inconsistent iff its unit equation bas solutions.

Proof: L to R: From our observations on consistent chains above, if a chain has a secondary matrix with a row of zeros, then it is consistent. So if it is inconsistent, then no row is all zeros. Hence by the lemma its unit equation has solutions. R to L: Conversely, if the unit equation has solutions, then by the lemma no row of $M_{s}$ is all zeros. But we also noted above that if a secondary matrix has no row all zeros, then it is inconsistent.

\subsection{DEGREE OF INCONSISTENCY}

The previous result motivates the following definition.

Definition i8 The Degree of inconsistency of a Necker chain is the number of independent solutions of its unit equation.

This seems intuitively reasonable: the unit equation tracks the independent ways in which both of the large faces get a non-zero value in the secondary equation. If the product of the secondary matrix with the column vector of crossings inevitably yields a zero face no matter what the state of the crossings, then it must have a row of zeros and so be consistent.

The test of the definition is in the results it produces, so we compute the degree of inconsistency for several salient cases.

(I) For individual Neckers, that is cells, we have that the trivial Necker has as its secondary matrix $\left[\begin{array}{ll}1 & 1 \\ 1 & 1\end{array}\right]$, which has solutions for $C=\left[\begin{array}{l}1 \\ 0\end{array}\right]$ and $\left[\begin{array}{l}0 \\ 1\end{array}\right]$. These are evidently independent of one another, so the number of independent solutions is at least 2 . It is at most 2 also, since there are no more independent solutions for $2 \times 1$ column vectors. Hence it is exactly 2 . This is the maximum degree of inconsistency for figures of this size, which accords with the intuition that the figure with red and blue at all crossings is maximally inconsistent.

(II) For the important case of the globally inconsistent Necker whose secondary matrix is Id, namely Escher's cube:

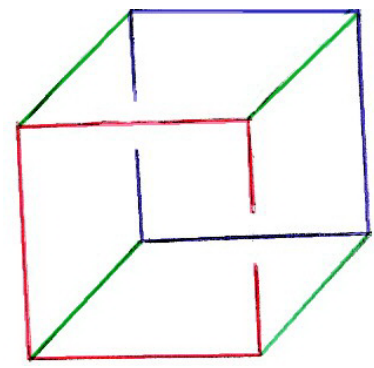

Figure 3: Global Inconsistency 
Its secondary matrix is $\left[\begin{array}{ll}1 & 0 \\ 0 & 1\end{array}\right]$, and we can compute that there is just one solution, $C=\left[\begin{array}{l}1 \\ 1\end{array}\right]$. Thus the degree of inconsistency is 1 , the intermediate value.

(III) For the consistent Neckers having at least one row of zeros, it is impossible to get solutions for the unit equation, so degree of inconsistency $=0$.

(Iv) The case $n=2$ (maximum independent solutions $=4$ ) contains more possibilities, and illustrates higher order inconsistency. For example, there is the example of the trivial Necker chain.

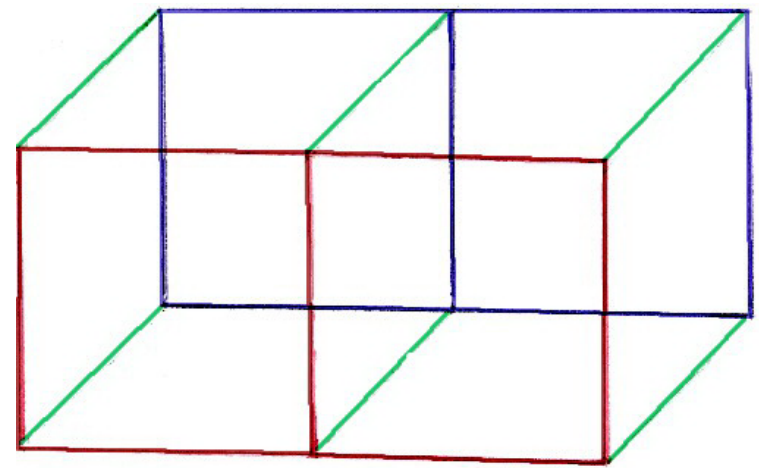

Figure 4: The Trivial 2-chain

This has solutions $\mathrm{C}=$ any of:

$$
\left[\begin{array}{l}
1 \\
0 \\
0 \\
0
\end{array}\right],\left[\begin{array}{l}
0 \\
1 \\
0 \\
0
\end{array}\right],\left[\begin{array}{l}
0 \\
0 \\
1 \\
0
\end{array}\right] \text { and }\left[\begin{array}{l}
0 \\
0 \\
0 \\
1
\end{array}\right]
$$

These solutions are all independent, so degree of inconsistency $=4$, which is maximal as triviality should be.

(v) Any chain with the same colour failing to appear at all crossings (ie. consistent), has a row of zeros and so has a degree of inconsistency $=0$, which is appropriate.

(vi) Any chain with both colours at a single crossing $C_{i}$ has a solution with the value for row $i=1$ and zeros elsewhere. Hence, with both colours at say 3 crossings, it has 3 independent solutions with a single 1 in that place, and has a degree of inconsistency $=3$. That is, adding inconsistent crossings increases the degree of inconsistency. 
(viI) Another important case is the diagram we began with, which displays higher order inconsistency. The secondary matrix for this figure is:

$$
\left[\begin{array}{llll}
1 & 1 & 0 & 0 \\
0 & 0 & 1 & 1
\end{array}\right]
$$

Its four solutions are:

$$
\left[\begin{array}{l}
1 \\
0 \\
0 \\
1
\end{array}\right],\left[\begin{array}{l}
0 \\
1 \\
1 \\
0
\end{array}\right],\left[\begin{array}{l}
1 \\
0 \\
1 \\
0
\end{array}\right] \text { and }\left[\begin{array}{l}
0 \\
1 \\
0 \\
1
\end{array}\right]
$$

However, each of these is the sum of the others, so any one can be eliminated. Hence, degree of inconsistency $=3$. Compare with Id above.

Conjecture: any n-chain whose cells are all copies of Id, (The Escher cube) has a degree of inconsistency $n$.

\section{$3 \cdot 3$ ELEMENTARY ROW AND COLUMN OPERATIONS}

We return to the properties of the Routley Functor and related operators. The results of the previous section cannot all remain true, because the primary and secondary matrices are not square, so that, for example, Transpose is not defined. Moreover, since matrices vary in size, operators must be defined differently for different sizes.

We begin with the three elementary row operations on the space of row vectors. First, RowSwaP ${ }_{i j}(M)$ is the result of swapping rows $i$ and $j$ in matrix $M$. We suppose that $M$ is $m \times n$. What matters for RowSwap is the number of rows $m$ on the matrix whose rows are being swapped. The basic swap operation is performed on $\mathrm{Id}_{m}$, which is the $m \times m$ square matrix with 1 down the main diagonal and zeros elsewhere. RowSwaP $\mathrm{P}_{i j}\left(\operatorname{Id}_{\mathrm{m}}\right)$ is thus the result of deleting the $1 \mathrm{~s}$ at places $(i, i)$ and $(j, j)$ and replacing them by $1 s$ at $(i, j)$ and $(j, i)$. It is then a simple matter to verify by matrix multiplication that $\operatorname{RowSwaP}_{i j}(M)$ can be achieved by left multiplication of $M$ by RowSwaP $P_{i j}\left(\operatorname{Id}_{m}\right)$.

The second elementary row operation is RowADD $1_{i j}$, which is the result of adding row $i$ to row $j$, and putting the result in row $i$. To define this, take $\mathrm{Id}_{m}$ and add a 1 in place $(i, j)=\operatorname{Id}_{m}+\left[a_{i j}=1\right]$. Then it is a simple matter to verify by matrix multiplication that left multiplication of $M$ by this matrix has the effect. The matrix is also the result of applying RowADD to Id itself. In the previous section, there is also distinguished RowADD $2_{i j}$, which adds row $i$ to row $j$ and puts the result in row $j$. But since RowADD $2_{i j}=\operatorname{RowADD} 1_{j i}$, this is not proceeded with.

The third elementary matrix operation is scalar multiplication. But since we remain in linear algebra over $Z_{2}$, then as in the single-cell Necker, the only nonzero scalar is 1 , so this operation is redundant. 
These can be summarised as:

THEOREM I9 (a) RowSwaP $\operatorname{Rij}_{i j}(M)=\operatorname{ROWSWAP}_{i j}(I d) \times M$

(b) $\operatorname{RowADD}_{i j}(M)=\operatorname{RowADD}_{i j}(I d) \times M=\left(I d+\left[a_{i j}=1\right]\right) \times M$

There are also elementary column operations ColSwap and ColAdd. They can also be defined in terms of (right) multiplication by a suitable matrix. In each case, the effect is obtained by performing the same operation on $\mathrm{Id}_{n}$, then right multiplication of $M$ by this. Note the index $n$ of $\operatorname{Id}_{n}$. This is necessary as right multiplication of a matrix $M$ which is $m \times n$, requires that Id have $n$ rows.

Then there is the useful and natural operation Sw ITCH, (not an elementary operation). This is the result of changing all os to is and versa. This effect can be obtained by adding $\mathbf{I}_{\mathrm{mn}}$, the $\mathrm{m} \times \mathrm{n}$ matrix of $1 \mathrm{~s}$. In summary:

THEOREM 20 (a) COLSwap $(M)=M \times \operatorname{CoLSwap}(I d)$

(b) $\operatorname{ColAdD}(M)=M \times \operatorname{ColAdD}(I d)$.

(c) $\operatorname{Switch}(M)=M+\mathbf{I}$

\subsection{THE ROUTLEY FUNCTOR REVISITED}

We recall that the Routley Functor interchanges rows $(1,1)$ for $(0,0)$, leaving rows $(0,1)$ and $(1,0)$ alone. Clearly then, in the general case, the Routley functor operates naturally only on matrices with 2 columns. Thus it operates on the primary matrix $M_{p}$, which is $2 n \times 2$.

In passing, we note that the effect of applying this operation to a chain of Neckers is to produce another chain, the chain obtained taking all crossings lacking any colour, and filling in with both colours, and taking any crossing with both colours and erasing both colours. That is, the Routley star is a natural transformation from Necker chains to Necker chains.

But it also has natural relationships with the above linear algebraic operators.

Theorem 2I (a) ${ }^{*}=$ Switch.ColSwap $=$ ColSwap.Switch

(b) Switch $={ }^{*}$.ColSwap $=$ ColSwap. ${ }^{*}$

(c) ColSwap $={ }^{*}$. Sw Itch $=$ Sw Itch. ${ }^{*}$

(d) ${ }^{*}=$ Switch.*.Switch

Proof: Ad (a): It is clear that operating on rows $(1,1)$ and $(0,0)$ in either order changes either into the other. On the other hand, operating on rows $(0,1)$ with ColSwap changes it to $(1,0)$ and then Switch changes it back. Similarly for the opposite order of operation. Similarly for operating on rows $(1,0)$ and $(0,1)$. Ad (b): $\operatorname{ColSwap}(1,1)=(1,1)$, and then ${ }^{*}(1,1)=(0,0)$ which 
is the effect of SwItch on the original. The other rows are similar. Ad (c): $\operatorname{SwITCH}(1,0)=(0,1)$, and then ${ }^{*}(0,1)=(0,1)$ which is the effect of ColSwap on the original. The other rows are similar. Ad (d): ${ }^{*}$ has no effect on rows $(0,1)$ and $(1,0)$, so the effect is two consecutive applications of SwIтCH, which is the identity. But for rows $(1,1)$ and $(0,0),{ }^{*}$ toggles between them, as does Switch.

We recall that there is also ANTI-* ${ }^{*}$, which does to columns what ${ }^{*}$ does to rows. This means that Anti-* only operates on matrices with two rows. With appropriate restrictions on the size of matrices then, we have, using similar calculations to the previous theorem:

Theorem 22 (a) Ant $i^{*}=$ Switch.RowSwap $=$ RowSwap.Switch

(b) Switch $=A n t i-i^{*} \cdot \operatorname{Row} S w a P=\operatorname{RowSwap} \cdot A n t i-{ }^{*}$

(c) RowSwap $=A n t i-{ }^{*} \cdot \operatorname{Switch}=\mathrm{Sw}$ ITCH.Anti-*

(d) $A n t i i^{*}=$ Switch.Anti- ${ }^{*}$.Sw I Tch

Note that we cannot put together part (b) of Theorems $2 \mathrm{I}$ and 22 to draw the conclusion as in Theorem IO that *.ColSwap $=$ Anti- ${ }^{*}$. RowSwap, because the size of the SwITch matrix is in general different on the LHS from the RHs.

However, we can say that the above results linking the Routley functor with other linear algebra operators remain true as generalisations of the results of the first section, and thus demonstrate the naturalness in this setting of the Routley functor and its dual, Anti-* .

\subsection{ORDER, COVARIANCE AND CONTRAVARIANCE}

Matrices of the same dimensions have the same definition of order as in the previous section, and the same arguments given there continue to hold. So we have:

THEOREM 23 (a) The following operators are covariant: RowSwAP, COLSwAP

(b) The following operators are contravariant: *, Anti-*, Sw IтCH.

Summing up this section: the main results are the description of chains of Neckers, the identification of higher-order inconsistencies arising from the impossible juxtaposition of consistent Necker cells, the definition of degree of inconsistency, and the method of calculation of degree of inconsistency as illustrated by a number of salient cases. In addition, it was seen that the Routley functor operates naturally on Necker chains, and is interdefinable in this context with other linear algebra operators. 


\section{CONCLUSION}

This paper demonstrates an application of linear algebra to the study of inconsistent and incomplete geometrical structures, the Necker cubes. We were able to show in the single-cell case that global and local consistency can be naturally classified together by matrix methods. Furthermore, when extending to chains of Neckers, it can be seen that the classification extends naturally to a kind of higher order inconsistency. The Routley Functor is shown to play a significant role in these classifications, and commutes with various linear-algebraic operations.

\section{REFERENCES}

[2006] mortensen, Chris, (2006), "An Analysis of Inconsistent and Incomplete Necker Cubes", The Australasian Fournal of Logic, 4, 216-225.

[2009] mortensen, Chris and steve leishman, (2009) "Linear Algebra Representation of Necker Cubes I: The Crazy Crate", The Australasian fournal of Logic, 7, I-9. 
The Australasian fournal of Logic (ISSN I448-5052) disseminates articles that significantly advance the study of logic, in its mathematical, philosophical or computational guises. The scope of the journal includes all areas of logic, both pure and applied to topics in philosophy, mathematics, computation, linguistics and the other sciences.

Articles appearing in the journal have been carefully and critically refereed under the responsibility of members of the Editorial Board. Only papers judged to be both significant and excellent are accepted for publication.

The journal is freely available at the journal website at

$$
\text { http://www.philosophy.unimelb.edu.au/ajl/ }
$$

All issues of the journal are archived electronically at the journal website.

SUBSCRIPTIONS Individuals may subscribe to the journal by sending an email, including a full name, an institutional affiliation and an email address to the managing editor at ajl-editors@unimelb.edu.au Subscribers will receive email abstracts of accepted papers to an address of their choice. For institutional subscription, please email the managing editor at ajl-editors@unimelb.edu.au

Complete published papers may be downloaded at the journal's website at http: //www.philosophy.unimelb.edu.au/ajl/ The journal currently publishes in pdf format.

SUBMISSION The journal accepts submissions of papers electronically. To submit an article for publication, send the $\mathrm{HT}_{\mathrm{E}} \mathrm{X}$ source of a submission to a member of the editorial board. For a current list of the editorial board, consult the website.

The copyright of each article remains with the author or authors of that article. 